\title{
Elusión fiscal (regulación en la Unión Europea) Tax avoidance (European Union regulation)
}

\author{
Rafael Sanz Gómez* \\ Universidad de Sevilla \\ rsanz@us.es
}

Recibido / received: 15/07/2017

Aceptado / accepted: 28/07/2017

DOI: https://doi.org/10.20318/eunomia.2017.3821

\begin{abstract}
Resumen
La elusión fiscal se define como la articulación de estrategias de minimización del pago de tributos que son conformes con la literalidad de la normativa pero no con una interpretación sistemática o teleológica, y ante las cuales el sistema tributario reacciona para, como mínimo, anular el beneficio fiscal obtenido. En el ámbito de la Unión Europea, el concepto de elusión como todo lo tributario- se analiza desde la perspectiva de la promoción de un mercado interior en condiciones de libre competencia. La postura de las instituciones de la Unión ha variado, pasando de favorecer ante todo la libre circulación de bienes, capitales, servicios y trabajadores, permitiendo la aplicación de las medidas anti-elusión estatales sólo con carácter excepcional, a considerar que la elusión daña las condiciones de libre competencia empresarial y es necesaria una acción positiva y coordinada, cuyo exponente más elaborado es la Directiva (UE) 2016/1164, del Consejo, de 12 de julio de 2016, por la que se establecen normas contra las prácticas de elusión fiscal que inciden directamente en el funcionamiento del mercado interior.
\end{abstract}

\section{Palabras clave}

Elusión fiscal, Unión Europea, abuso del derecho tributario, planificación fiscal agresiva, mercado interior, BEPS, armonización.

\footnotetext{
Abstract

Tax avoidance is defined as the implementation of strategies of minimization of the tax burden that are in accordance with the literal wording of the regulations but not with their systematic or teleological interpretation. The tax system reacts to tax avoidance by, at least, supressing the benefit obtained by the taxpayer. Within the European Union, the concept of tax avoidance -like everything regarding tax matters-is analysed from the perspective of the promotion of an internal market under conditions of free competition. The stand taken by the Union institutions has shifted from promoting free movement of goods, capital, services, and labour while allowing the application of State anti-avoidance measures only on an exceptional basis, to consider that tax avoidance damages the conditions of free competition and positive

*Profesor Ayudante Doctor, Universidad de Sevilla.

El autor agradece los comentarios y sugerencias de las dos personas anónimas que han realizado la revisión del trabajo original. Todo error o inexactitud es achacable exclusivamente al autor.
} 
and coordinated action is necessary. Here, a milestone has been the enaction of the Council Directive (EU) 2016/1164 of 12 July 2016 laying down rules against tax avoidance practices that directly affect the functioning of the internal market.

\title{
Keywords
}

Tax avoidance, European Union, tax abuse, aggressive tax planning, internal market, BEPS, harmonization.

\begin{abstract}
SUMARIO. 1. Introducción. La elusión fiscal como "zona gris". 2. La elusión fiscal como límite -excepcional- a las libertades de circulación. 3. La elusión fiscal como fenómeno distorsionador de las condiciones de libre competencia en el mercado interior. 4. A modo de conclusión.
\end{abstract}

\section{Introducción. La elusión fiscal como "zona gris"}

El término "elusión fiscal" se refiere al uso de estrategias de minimización del pago de tributos mediante mecanismos que, aunque no infringen de manera abierta la normativa, sí reciben un reproche del ordenamiento jurídico, como mínimo la anulación del ahorro fiscal obtenido. La Comisión de Asuntos Económicos y Monetarios del Parlamento Europeo ha definido la elusión como "una utilización legal pero indebida del régimen tributario para reducir o evitar responsabilidades fiscales"1. Es tradicional diferenciar entre fraude o evasión fiscal (reducción o evitación del pago mediante una violación abierta de la norma), elusión fiscal (que, como vemos, pertenece a una "zona gris" en cuanto a su conformidad con el ordenamiento jurídico) y planificación fiscal legítima o economía de opción (supuestos admisibles de reducción de la deuda tributaria). El ordenamiento reacciona ante la elusión porque considera que las operaciones realizadas son contrarias al espíritu o finalidad de las normas o porque existe una discrepancia entre la forma de las operaciones realizadas y su realidad económica. En el ámbito internacional, la elusión suele implicar el aprovechamiento indebido de las diferencias de tributación derivadas de los beneficios fiscales previstos por otros Estados, lo cual sólo puede solventarse con mayor coordinación entre jurisdicciones y la erradicación de prácticas de competencia fiscal perniciosa (Lucas Durán, 2014, p. 616; García Novoa, 2015, p. 38).

Pistone (2016) afirma que "el fenómeno de la elusión fiscal se concreta en función de diversos matices, según sea la técnica utilizada por cada ordenamiento para determinar los límites dentro de los que se toleran los fenómenos de ahorro fiscal". Los instrumentos con que se combate la elusión, puesto que determinan qué manifestaciones de planificación fiscal son aceptables (economía de opción) y cuáles no lo son (elusión fiscal), terminan de definir esta última. Pueden diferenciarse dos grandes grupos de instrumentos anti-elusión: las cláusulas específicas, que son normas que se dirigen a supuestos concretos, ante los que establecen hechos imponibles complementarios, presunciones o auténticas ficciones legales; y las cláusulas generales, potencialmente aplicables a cualquier supuesto, siempre y cuando se cumpla un presupuesto de hecho formulado en términos amplios.

Para entender el tratamiento de la elusión en el Derecho de la Unión debemos partir del hecho de que la Unión Europea tiene unas competencias

\footnotetext{
${ }^{1}$ Informe de 03-05-2013 sobre la lucha contra el fraude fiscal, la evasión fiscal y los paraísos fiscales $(2013 / 2060($ INI)).
} 
limitadas en el ámbito tributario. Tales competencias responden siempre a razones extrafiscales (Casado Ollero, 1985, p. 347), fundamentalmente la creación de un espacio sin fronteras interiores para la libre circulación de personas, servicios, mercancías y capitales. En el ámbito de la imposición indirecta, el art. 113 del Tratado de Funcionamiento de la Unión Europea (TFUE) permite al Consejo, por unanimidad, a iniciativa de la Comisión y previa consulta no vinculante al Parlamento Europeo, adoptar disposiciones de armonización de las legislaciones estatales "en la medida en que dicha armonización sea necesaria para garantizar el establecimiento y el funcionamiento del mercado interior y evitar las distorsiones de la competencia". En relación a la imposición directa, el art. 115 TFUE permite adoptar disposiciones de armonización por un procedimiento equivalente y con la misma subordinación a las necesidades del mercado interior y la libre competencia. Por lo demás, y ante las dificultades tradicionales para obtener la unanimidad requerida por los arts. 114 y 115 TFUE, durante mucho tiempo los vectores de cambio de los sistemas tributarios de los Estados miembros han sido la jurisprudencia del Tribunal de Justicia de la Unión Europea (en lo sucesivo, TJUE), quien ha aplicado las libertades de circulación de los Tratados para suprimir medidas restrictivas o discriminatorias, y el régimen de ayudas de Estado (arts. 107 a 109 TFUE), que la Comisión ha empleado para suprimir los beneficios fiscales selectivos que, al apoyar a empresas residentes, distorsionasen la competencia.

El concepto de "elusión fiscal" y su tratamiento en la UE ha ido modificándose a medida que se reconfiguraba la relación entre prácticas elusivas y competencia empresarial. Se alcanza un punto de inflexión al consolidarse la idea de que es necesario actuar frente a las actuaciones elusivas para garantizar la igualdad de condiciones entre empresas: las empresas que eluden resultan más competitivas que las que cumplen si no hay una reacción de los poderes públicos; además, las empresas transnacionales tienen acceso a mecanismos de elusión fiscal fuera del alcance de las pymes.

A nivel estrictamente terminológico, cabe señalar algunas cuestiones preliminares. Puesto que los conceptos empleados en cada Estado para describir la elusión eran ( $\mathrm{y}$ en parte siguen siendo, pese a cierta aproximación) distintos, y lo mismo sucede con los instrumentos empleados para combatirla, todavía hoy la doctrina advierte que se usan términos diferentes como si fueran sinónimos, sin serlo (Cordewener, 2017, p. 61). En el caso del castellano, se da además la circunstancia de que en la normativa de la Unión y en la jurisprudencia del TJUE se ha venido usando erróneamente el término "fraude" donde debía emplearse "elusión" (probablemente por influencia del francés, donde "évasion" no significa evasión, sino elusión).

\section{La elusión fiscal como límite-excepcional-a las libertades de circulación}

Las principales directivas en materia tributaria que se han dictado contienen disposiciones que habilitan a los Estados miembros a no aplicar el régimen correspondiente cuando sea necesario para prevenir la elusión o la evasión fiscal. Así se señalaba en la Sexta Directiva sobre IVA ${ }^{2}$, de 1977, y se mantiene en la Directiva 2006/112/CE vigente ${ }^{3}$; también en las distintas versiones de la Directiva

\footnotetext{
2 Sexta Directiva 77/388/CEE del Consejo, de 17-05-1977, en materia de armonización de las legislaciones de los Estados Miembros relativas a los impuestos sobre el volumen de negocios Sistema común del Impuesto sobre el Valor Añadido: base imponible uniforme.

${ }^{3}$ Directiva 2006/112/CE del Consejo, de 28-11-2006, relativa al sistema común del impuesto sobre el valor añadido.
} 
sobre operaciones de reorganización societaria ${ }^{4}$ y la Directiva matriz-filial ${ }^{5}$; y en la Directiva sobre intereses y cánones ${ }^{6}$. La incorporación del término "elusión" a estas directivas lo convierte en un concepto de Derecho de la Unión, cuya definición queda fuera del alcance de los Estados miembros y corresponde al TJUE ${ }^{7}$. Por otra parte, según la jurisprudencia del Tribunal, los beneficios reconocidos en la normativa de la Unión sólo pueden denegarse en supuestos excepcionales, por lo que los términos de las cláusulas habilitantes de las Directivas deben ser objeto de interpretación estricta.

En ámbitos no armonizados, el TJUE también limitó las condiciones bajo las cuales los Estados podían mantener cláusulas específicas anti-elusión. Las primeras sentencias no excluyeron de raíz la posibilidad de establecer estas cláusulas, pero deberían estar justificadas por razones imperiosas de interés general y ser proporcionadas $^{8}$. Las razones de interés general admitidas por el Tribunal son, fundamentalmente, dos: el reparto equilibrado de la potestad impositiva y, sobre todo, la prevención de conductas abusivas.

En ámbitos no armonizados, el concepto de abuso se construye sobre la noción de "montajes puramente artificiales", que aparece por primera vez en la sentencia Imperial Chemical Industries ${ }^{9}$. Este término procede de una interpretación teleológica de las libertades fundamentales y determina cuándo un particular no queda bajo la protección de la libertad fundamental invocada porque en realidad no estaría realizando una actividad económica sustancial. Así, la lucha contra la elusión fiscal se presenta como una cuestión secundaria, permisible en la medida en que las libertades fundamentales de los Tratados de la UE (que tienen primacía sobre el Derecho estatal) no puedan invocarse con éxito por los particulares. Un montaje puramente artificial, en el ámbito del derecho de establecimiento, describe una entidad que no cumple con unos criterios mínimos de implantación en el Estado en que opera, de medios personales y materiales disponibles, de funciones atribuidas y de adición de valor a la cadena productiva. Las sentencias Cadbury Schweppes ${ }^{10} \mathrm{o}$ Test Claimants in the Thin Cap Group Litigation ${ }^{11}$ consolidaron este concepto y fueron marcando un desplazamiento de la jurisprudencia del Tribunal hacia posiciones más favorables al interés fiscal de los Estados (Ruiz Almendral, 2013, p. 157). Todas las sentencias citadas hacen referencia a la libertad de establecimiento, en torno a la cual se construye esta noción, aunque se traslada luego a las demás libertades. En general, una estructura (entidad, operación, contrato) constituirá un

\footnotetext{
${ }^{4}$ Directiva 90/434/CEE del Consejo, de 23-07-1990, relativa al régimen fiscal común aplicable a las fusiones, escisiones, aportaciones de activos y canjes de acciones realizados entre sociedades de diferentes Estados miembros; actualmente, la Directiva 2009/133/CE del Consejo, de 19-10-2009, relativa al régimen fiscal común aplicable a las fusiones, escisiones, escisiones parciales, aportaciones de activos y canjes de acciones realizados entre sociedades de diferentes Estados miembros y al traslado del domicilio social de una SE o una SCE de un Estado miembro a otro.

5 Directiva 90/435/CEE del Consejo, de 23-07-1990, relativa al régimen fiscal común aplicable a las sociedades matrices y filiales de Estados Miembros diferentes; actualmente, la Directiva 2011/96/UE del Consejo, de 30-11-2011, relativa al régimen fiscal común aplicable a las sociedades matrices y filiales de Estados miembros diferentes (refundición).

6 Directiva 2003/49/CE del Consejo, de 03-06-2003, relativa a un régimen fiscal común aplicable a los pagos de intereses y cánones efectuados entre sociedades asociadas de diferentes Estados miembros.

Sentencia de 12-07-1988, Direct Cosmetics y Laughtons Photographs, 138/86 y 139/86, ECLI:EU:C:1988:383, ap. 20. El apartado citado refleja el error de traducción que señalamos en el epígrafe introductorio de este trabajo, al hablar de "evasión" (en inglés se usa "avoidance").

8 Sentencia de 12-12-2002, Lankhorst-Hohorst, C-324/00, ECLI:EU:C:2002:749.

${ }^{9}$ Sentencia de 16-07-1998, Imperial Chemical Industries, C-264/96, ECLI:EU:C:1998:370.

10 Sentencia de 12-09-2006, Cadbury Schweppes y Cadbury Schweppes Overseas, C-196/04, ECLI:EU:C:2006:544.

11 Sentencia de 13-03-2007, Test Claimants in the Thin Cap Group Litigation, C-524/04, ECLI:EU:C:2007:161.
} 
"montaje puramente artificial" cuando, sobre la base de criterios objetivos, pueda determinarse que no es expresión de un ejercicio real o sustancial de la libertad invocada.

Según el TJUE, las cláusulas anti-elusión estatales deben afectar exclusivamente a montajes puramente artificiales. Los Estados pueden establecer criterios que sirvan como indicios de conducta abusiva, pero se debe siempre reconocer al particular la opción de demostrar que la actividad realizada tiene un sustrato económico real. La consecuencia de la aplicación de estas cláusulas suele ser la recalificación de las operaciones realizadas a la luz de los negocios que se emplearían habitualmente para obtener el resultado efectivamente alcanzado.

En las materias armonizadas, la jurisprudencia del Tribunal se basa en la sentencia Emsland-Stärke ${ }^{12}$, dictada en relación con una cuestión aduanera y que a su vez toma como referencia el Reglamento (CE, Euratom) núm. 2988/95 del Consejo, de 18 de diciembre de 1995, relativo a la protección de los intereses financieros de las Comunidades Europeas. Pasa al ámbito del IVA a través de sentencias como Halifax ${ }^{13}$ o Part Service ${ }^{14}$. A la luz de esta jurisprudencia, se considerará abusiva la invocación formal de una norma para obtener una ventaja fiscal cuya concesión sería contraria al objetivo perseguido por tal norma cuando, además, se observe que el objetivo esencial de las operaciones consista en obtener una ventaja fiscal (ponderando la relevancia de dicha finalidad con la existencia de una lógica económica en la operación). Cabe destacar que, al ser el IVA un recurso propio de la Unión Europea, el TJUE ha declarado que combatir la elusión de este impuesto es un deber de los Estados.

Las dos líneas jurisprudenciales se han ido aproximando, con referencias cruzadas entre ellas; dando lugar, para parte de la doctrina, a un principio anti-abuso único (Martín Jiménez, 2012, pp. 278-280; Sanz Gómez, 2012, pp. 226-235) que se aplica tanto en los ámbitos armonizados como en los no armonizados. El principio de abuso constituye un límite intrínseco de los derechos reconocidos por el Derecho de la Unión. Se define sobre la base de dos elementos, ambos de carácter objetivo: el ejercicio de un derecho o la aplicación de una norma de forma contraria a su finalidad y la organización de actividades u operaciones de modo que la consecución de un ahorro fiscal prevalezca sobre las lógicas económicas.

La interrelación entre elusión fiscal y abuso es tan grande que parte de la doctrina afirma que ambos términos pueden considerarse sinónimos. Por ejemplo, para Zalasiński (2008, p. 160), hacen referencia al mismo tipo de comportamiento de los contribuyentes y pueden contemplar las mismas situaciones fácticas. Cordewener (2017, p. 61) señala que pueden ser conceptos equivalentes o que guarden una relación de género y especie. Si nos remitimos a su uso estricto en el ámbito de la Unión Europea, el concepto de abuso parece ser más limitado puesto que, junto al principio anti-abuso, el ordenamiento de la Unión Europea ha admitido la lucha contra la elusión con el objetivo de garantizar un reparto equilibrado de la potestad tributaria entre Estados. Este "reparto equilibrado", además de una razón de interés general que puede justificar una medida estatal discriminatoria o restrictiva (Ruiz Almendral, 2013), es una idea consustancial a la aprobación de Directivas en materia tributaria o convenios contra la doble imposición entre Estados; y, como veremos en el apartado siguiente, es el criterio en que se basan algunas de las medidas de la Directiva anti-elusión de 2016.

\footnotetext{
${ }^{12}$ Sentencia de 14-12-2000, Emsland-Stärke, C-110/99, ECLI:EU:C:2000:695.

${ }^{13}$ Sentencia de 21-02-2006, Halifax y otros, C-255/02, ECLI:EU:C:2006:121.

${ }^{14}$ Sentencia de 21-02-2008, Part Service, C-425/06, ECLI:EU:C:2008:108.
} 
3. La elusión físcal como fenómeno distorsionador de las condiciones de libre competencia en el mercado interior

La preocupación de las instituciones de la Unión Europea por prevenir la elusión y la evasión en el mercado interior mereció, en un primer momento, la calificación de "tímida" (Lucas Durán, 2014, p. 624). A partir de 2012 sí se observa un cambio de tendencia, con el lanzamiento de un Plan de Acción para reforzar la lucha contra el fraude fiscal y la evasión fiscal ${ }^{15}$. Se ha afirmado que el precedente más inmediato de esta política es, precisamente, la jurisprudencia del TJUE sobre el concepto de abuso (García Novoa, 2015, p. 44) que hemos analizado. El Plan es inseparable del Proyecto BEPS de la OCDE: "BEPS" es el acrónimo en inglés para Base Erosion and Profit Shifting, y hace referencia a la erosión de bases imponibles y al traslado de beneficios que consiguen las empresas transnacionales aprovechando lagunas 0 desajustes entre distintos sistemas impositivos estatales. En gran medida, es fruto de la discordancia entre los modos de producción global propios de las empresas transnacionales y un sistema tributario internacional basado en los convenios bilaterales contra la doble imposición ${ }^{16}$.

Aunque el concepto de "elusión" no estaba presente en el documento que describía el Plan de Acción de la Comisión, sí se hacía referencia a la "planificación fiscal agresiva" que, según la Recomendación de la Comisión de 6 de diciembre de 2012, sobre planificación fiscal agresiva (2012/772/UE), equivale a una de las definiciones de elusión que venimos manejando: "mecanismos que, pese a ser estrictamente legales, son contrarios al espíritu de la ley".

Sin embargo, la aparición del concepto "planificación fiscal agresiva" dificulta más aún la tarea de definir con claridad la elusión fiscal. Aquel concepto se encontraba ya en el Estudio sobre intermediarios fiscales de la OCDE de 2008, para hacer referencia a decisiones de planificación fiscal difícilmente defendibles ante un Tribunal. Posteriormente ha ido evolucionando, en el ámbito de la OCDE, para hacerse eco de las líneas del proyecto BEPS. Así, el concepto de planificación fiscal agresiva se aproxima al de elusión mediante operaciones que aprovechan las asimetrías entre ordenamientos tributarios estatales para desplazar artificiosamente los beneficios a jurisdicciones con una baja o nula presión fiscal, algo en la órbita de lo que para parte de la doctrina es la elusión fiscal en el ámbito internacional. Según Pistone (2016, p. 130), existe cierto solapamiento entre los conceptos y "la línea de separación [...] está rodeada de un áurea de incertidumbre". Cordewener (2017, p. 61) afirma que el término de planificación fiscal agresiva es tan amplio que puede abarcar tanto la evasión como la elusión fiscal, aunque en la práctica muchas veces equivale a abuso. Por último, otros autores -es la línea que consideramos más acertada- afirman que el concepto de "planificación fiscal agresiva" pertenece al ámbito del soft law y se refiere no a la legalidad de la planificación fiscal sino a su legitimidad (Panayi, 2015).

Dentro de la estrategia contra la elusión fiscal que la Unión Europea despliega desde 2012 se observa también un uso más intenso del régimen de ayudas de Estado. Existe un vínculo relevante entre la competencia fiscal perniciosa (de la que las ayudas de Estado pueden ser una manifestación) y la elusión fiscal, en la medida en que la primera crea oportunidades para la segunda. La Comisión ha

\footnotetext{
${ }^{15}$ Comunicación de la Comisión $\operatorname{COM}(2012) 722$ final, de 06-12-2012.

${ }^{16}$ La OCDE publicó un informe preliminar en febrero de 2013 y un Plan de Acción en julio de 2013. El Plan de Acción se divide en quince acciones específicas que se han ido elaborando entre 2013 y 2015.
} 
impulsado un proceso de modernización del régimen de ayudas de Estado ${ }^{17}$, que incluyó una reforma de los cauces procedimentales ${ }^{18}$ y nuevas directrices para definir el concepto de ayuda ${ }^{19}$.

Finalmente, la reacción más específica de la Comisión ante el fenómeno BEPS parte de lo establecido en la Comunicación $\operatorname{COM(2015)~} 302$ final, de 17 de junio de 2015, al Parlamento Europeo y al Consejo, titulada "Un sistema de imposición de las sociedades justo y eficaz en la Unión Europea: cinco ámbitos de actuación fundamentales". Su desarrollo, en la lucha contra la planificación fiscal agresiva, se concretó en el llamado paquete anti-elusión fiscal ("Anti Tax Avoidance Package"), que contenía la propuesta de Directiva que luego sería aprobada como Directiva (UE) 2016/1164 del Consejo, de 12 de julio de 2016, por la que se establecen normas contra las prácticas de elusión fiscal que inciden directamente en el funcionamiento del mercado interior; y que ha sido modificada por la Directiva (UE) 2017/952 del Consejo, de 29 de mayo de 2017, en lo que se refiere a las asimetrías híbridas con terceros países.

La Directiva (UE) 2016/1164 es una norma con un significado complejo. En su preámbulo se define como una reacción ante las necesidades puestas de manifiesto por el proyecto BEPS; pero hay voces críticas que señalan que es una iniciativa contraria al espíritu de multilateralismo que inspira BEPS y que contiene elementos no previstos en dicho proyecto, como un impuesto de salida o una cláusula general anti-abuso (Calderón Carrero y Martín Jiménez, 2017, p. 50). La Directiva también apela a la necesidad de "evitar la fragmentación del mercado y poner fin al falseamiento del mercado y a las asimetrías existentes en la actualidad". Detectamos de nuevo la idea de que la elusión fiscal tiene un impacto negativo sobre la libre competencia, hasta el punto que "un nivel mínimo de protección frente a las prácticas de elusión fiscal en los sistemas nacionales del impuesto sobre sociedades en toda la Unión" implica también "establecer un nivel mínimo común de protección del mercado interior en sectores específicos" 20 .

La Directiva contiene una norma relativa a la limitación de deducibilidad de los intereses intragrupo, un impuesto de salida, una cláusula general anti-abuso, una norma sobre sociedades extranjeras controladas y una serie de reglas sobre asimetrías híbridas. Aunque no podemos analizarlas en detalle por falta de espacio, sí nos permiten confirmar que la UE combate la elusión a través del principio antiabuso y de criterios de reparto de la potestad tributaria entre los Estados miembros. Todas las disposiciones aprobadas se sustentan en una u otra justificación, e incluso encontramos diferentes posturas doctrinales sobre el fundamento de alguna de las cláusulas. La Directiva sólo afecta a las conductas elusivas que se produzcan en el ámbito de los impuestos sobre sociedades y convierte el combate a la elusión en un deber de los Estados, no un derecho como hasta entonces ${ }^{21}$.

La relación entre la Directiva anti-elusión y la doctrina del TJUE que desarrolla las libertades de circulación y el abuso de las mismas es compleja y no cabe descartar que se declare alguna violación de los Tratados por parte del legislador de la Unión. A título de ejemplo, hay autores (Os, 2016; Dourado, 2017)

\footnotetext{
${ }^{17}$ Comunicación de la Comisión COM (2012) 209 final, de 08-05-2012. Modernización de las ayudas estatales en la UE.

${ }^{18}$ Reglamento (UE) núm. 734/2013 del Consejo, de 22-07-2013, que modifica el Reglamento (CE) núm. 659/1999 por el que se establecen disposiciones de aplicación del artículo 93 del Tratado CE.

${ }^{19}$ Comunicación de la Comisión relativa al concepto de ayuda estatal conforme a lo dispuesto en el artículo 107, apartado 1, del Tratado de Funcionamiento de la Unión Europea (2016/C 262/01).

${ }^{20}$ Todos los fragmentos entrecomillados son del preámbulo de la Directiva.

${ }^{21}$ Sí era ya un deber de los Estados combatir la elusión en el ámbito del IVA.
} 
que han apuntado que la norma relativa a la limitación de intereses de la Directiva puede constituir una restricción desproporcionada de las libertades fundamentales. En ámbitos donde la armonización es limitada y deja un evidente margen de discrecionalidad para los Estados, la Directiva se interpretará de acuerdo con los Tratados y el TJUE analizará en la práctica si la legislación nacional es conforme con las libertades de circulación ${ }^{22}$. No está tan claro, sin embargo, el grado de discrecionalidad de que dispone el Consejo cuando dicta Directivas que contienen reglas muy detalladas, como es el caso; aunque lo cierto es que es muy raro que la normativa derivada se declare contraria a los Tratados (Caro de Sousa, 2015, p. 119). Parece que el legislador puede tener en consideración cuestiones (por ejemplo, razones presupuestarias) que el TJUE ha rechazado de manera sistemática como justificación para que un Estado, unilateralmente, limite las libertades fundamentales. También se ha afirmado que el principio de proporcionalidad se aplica menos estrictamente sobre actos de instituciones de la UE (Caro de Sousa, 2015, p. 48). Os (2016, p. 196) resume el estado de la cuestión afirmando que el alcance de la discrecionalidad del legislador de la Unión es una problemática que "ha sido planteada, pero no resuelta" por la doctrina. Por razones de extensión, tampoco se podrá analizar con mayor profundidad en este trabajo.

\section{A modo de conclusión}

El concepto de elusión fiscal en el Derecho de la Unión Europea se construye a través de un diálogo entre los agentes del sistema jurídico (Estados, Comisión Europea, Consejo, Tribunal de Justicia, particulares) y puede extraerse de un análisis de dicho diálogo. La elusión fiscal se refiere, con carácter general, a aquellas operaciones o actividades tendentes a reducir el pago de tributos y que, aunque respetan la literalidad de la normativa aplicable, no son respetuosas con el ordenamiento jurídico interpretado desde una perspectiva sistémica y con base en criterios teleológicos. Hemos concretado esta definición a través del análisis de la aceptación o rechazo de las cláusulas anti-elusión estatales por parte del TJUE y de las normas contra la elusión fiscal creadas en el ámbito de la propia Unión.

A medida que se reajustaban los intereses en juego, la postura de las instituciones de la UE ha ido variando desde una promoción preferente de las libertades de circulación en el mercado interior hacia una constatación de que las condiciones de libre competencia se ponen en riesgo si no hay una acción decidida contra la elusión fiscal. Ello se pone de manifiesto en un cambio de tendencia en la doctrina del TJUE desde 2005, en mayores esfuerzos de coordinación por parte de la Comisión y un avance sin precedentes en la producción normativa anti-elusión, cuyo elemento más destacado es la Directiva (UE) 2016/1164.

De la doctrina del Tribunal y del contenido de la Directiva (UE) 2016/1164 se extrae que existen fundamentalmente dos razones que justifican la aplicación de medidas anti-elusión: la prohibición de abuso y la defensa de la integridad del sistema tributario. La doctrina ha afirmado que algunos de los preceptos de la Directiva podrían ser contrarios a los Tratados por establecer restricciones desproporcionadas a la libertad de establecimiento. Para determinar si es así, será necesario aclarar previamente dos cuestiones que no se han resuelto satisfactoriamente aún y que están estrechamente relacionadas: cuál es la relación entre el Derecho originario y el derivado, y cuál es el margen de discrecionalidad de que dispone el Consejo en el ejercicio de su función legislativa frente a las libertades de circulación.

\footnotetext{
${ }^{22}$ Sentencia de 18-09-2003, Bosal, C-168/01, ECLI:EU:C:2003:479.
} 
Bibliografía

CALDERÓN CARRERO, J. M., y MARTíN JIMÉNEZ, A. (2017), "La Directiva UE 2016/1164 contra las prácticas de elusión fiscal que inciden en el mercado interior: ¿El principio del final de un IS nacional o una pieza más de descoordinación en el nuevo orden post-BEPS?", Estudios financieros. Revista de contabilidad y tributación, núm. 407, pp. 5-56.

CARO DE SOUSA, P. (2015), The European Fundamental Freedoms. A Contextual Approach. Oxford University Press, Oxford.

CASADO OLLERO, G. (1985), "Extrafiscalidad e incentivos fiscales a la inversión en la Comunidad Económica Europea", Hacienda Pública Española, núm. 96, pp. 333-372.

CORDEWENER, A. (2017), "Anti-Abuse Measures in the Area of Direct Taxation: Towards Converging Standards under Treaty Freedoms and EU Directives?", EC Tax Review, núm. 2, pp. 60-66.

DOURADO, A. P. (2017), "The Interest Limitation Rule in the Anti-Tax Avoidance Directive (ATAD) and the Net Taxation Principle", EC Tax Review, núm. 3, pp. 112-121.

DURÁN-SINDREU BUXADÉ, A. (2007), Los Motivos Económicos Válidos como Técnica Contra la Elusión Fiscal: Economía de Opción, Autonomía de Voluntad y Causa en los Negocios, Aranzadi, Cizur Menor (Navarra).

GARCÍA NOVOA, C. (2015), "Harmonización e loita contra a elusión tributaria na Unión Europea", Administración \& cidadanía. Revista da Escola Galega de Administración Pública, vol. 10, núm. 1, pp. 35-64.

LUCAS DURÁN, M. (2014), "Coordinación en el seno de la Unión Europea para luchar contra el fraude y elusión fiscales. Viejas y nuevas medidas". En: ADAME MARTÍNEZ, F. J. y RAMOS PRIETO, J., Estudios sobre el sistema tributario actual y la situación financiera del sector público: Homenaje al Profesor Dr. D. Javier Lasarte Álvarez, Madrid, Instituto de Estudios Fiscales, pp. 615-643.

MARTÍN JIMÉNEZ, A. (2012), "Towards a Homogeneous Theory of Abuse in EU (Direct) Tax Law", Bulletin for International Taxation, vol. 66, núm. 4/5, pp. 270292.

OS, P. (2016), "Interest Limitation under the Adopted Anti-Tax Avoidance Directive and Proportionality". EC Tax Review, núm. 4, pp. 184-198.

PANAYI, C. H. (2015), "Is Aggressive Tax Planning Socially Irresponsible?", Intertax, vol. 43, núm. 10, pp. 544-558

PISTONE, P. (2016), "La planificación fiscal agresiva y las categorías conceptuales del derecho tributario global", Revista Española de Derecho Financiero, núm. 170 , pp. $109-151$.

RUIZ ALMENDRAL, V. (2013), "Tax Avoidance, the 'Balanced Allocation of Taxing Powers' and the Arm's Length Standard: An Odd Threesome in Need of Clarification". En: RICHELLE, I., SCHÖN, W., y TRAVERSA, E., Allocating Taxing Powers within the European Union. MPI Studies in Tax Law and Public Finance, vol. 2, Heidelberg, Springer, pp. 131-170.

SANZ GÓMEZ, R. (2012), La lucha de los Estados contra la elusión fiscal en el contexto de la Unión Europea, Civitas, Cizur Menor (Navarra).

ZALASIŃSKY, A. (2008), "Some Basic Aspects of the Concept of Abuse in the Tax Case Law of the European Court of Justice". Intertax, vol. 36, núm. 4, pp. 156167. 